\title{
Frequent utilization of the emergency department for acute exacerbation of chronic obstructive pulmonary disease
}

\author{
Kohei Hasegawa ${ }^{1,2^{*}}$, Yusuke Tsugawa ${ }^{3}$, Chu-Lin Tsai ${ }^{1,2}$, David FM Brown ${ }^{1,2}$ and Carlos A Camargo Jr $r^{1,2}$
}

\begin{abstract}
Background: Little is known about patients who frequently visit the emergency department (ED) for acute exacerbation of chronic obstructive pulmonary disease (AECOPD). We aimed to quantify the proportion and characteristics of patients with frequent ED visits for AECOPD and associated healthcare utilization.

Methods: We conducted a retrospective cohort study of adults aged $\geq 40$ years with at least one ED visit for AECOPD between 2010 and 2011, derived from population-based all-payer data of State ED and Inpatient Databases for two large and diverse states: California and Florida. Outcome measures were frequency of ED visits for AECOPD, 30-day ED revisits, subsequent hospitalizations, near-fatal events (AECOPD involving mechanical ventilation), and charges for both ED and inpatient services (available only for Florida) during the year after the first ED visit.

Results: The analytic cohort comprised 98,280 unique patients with 154,736 ED visits for AECOPD. During the 1-year period, $29.4 \%$ (95\% Cl, 29.1\%-29.7\%) of the patients had two or more (frequent) visits, accounting for 55.2\% (95\% Cl, 54.9\%-55.4\%) of all ED visits for AECOPD. In the multivariable model, significant predictors of frequent ED visits were age 55-74 years (vs. 40-54 years), male sex, non-Hispanic white or black race, Medicaid insurance (vs. private), and lower median household income (all P < 0.001). At the visit-level, 12.3\% of ED visits for AECOPD were 30-day revisit events ( $95 \% \mathrm{Cl}, 12.1 \%-12.4 \%)$. Additionally, 62.8\% of ED visits for AECOPD ( $95 \% \mathrm{Cl}, 62.6 \%-63.0 \%)$ resulted in a hospitalization; patients with frequent ED visits comprised 55.5\% (95\% Cl, 55.2\%-55.8\%) of all hospitalizations. Furthermore, $7.3 \%$ (95\% Cl, 7.3\%-7.5\%) of ED visits for AECOPD led to a near-fatal event; patients with frequent ED visits accounted for $64.4 \%$ (95\% Cl, 63.5\%-65.3\%) of all near-fatal events. Total charges for AECOPD were $\$ 1.94$ billion (95\% Cl, \$1.90-1.97 billion) in Florida; patients with frequent ED visits accounted for \$1.07 billion (95\% Cl, \$1.04-1.09 billion).
\end{abstract}

Conclusions: In this large cohort study, we found that 29\% had frequent ED visits for AECOPD and that lower socioeconomic status was significantly associated with a higher frequency of ED visits. Individuals with frequent ED visits for AECOPD accounted for a substantial amount of healthcare utilization and financial burden.

Keywords: Chronic obstructive pulmonary disease, Exacerbation, Recurrence, Epidemiology, Emergency service, Hospitalization, Mechanical ventilation, Cost, Healthcare utilization

\footnotetext{
*Correspondence: khasegawa1@partners.org

${ }^{1}$ Department of Emergency Medicine, Massachusetts General Hospital, 326

Cambridge Street, Suite 410, Boston, MA 02114, USA

${ }^{2}$ Harvard Medical School, Boston, MA, USA

Full list of author information is available at the end of the article
} 


\section{Background}

Chronic obstructive pulmonary disease (COPD) is a serious public health problem in the US. Acute exacerbations of COPD (AECOPD) were responsible for approximately 1.5 million emergency department (ED) visits and 700,000 hospitalizations in 2010 [1]. Furthermore, acute exacerbations accelerate decline in lung function, reduce patient's quality of life, and increase health care use [2-4]. Indeed, exacerbation management was estimated to account for up to $70 \%$ of total direct costs of COPD management [5,6].

In this context, the US government recently identified reducing ED visits for AECOPD as a national objective in Healthy People 2020 [7]. To develop and implement preventive strategies effectively, identifying patients at risk for future exacerbations is critical. Prior studies have identified risk factors associated with frequent exacerbations ( 2 or more exacerbations in a year), such as a history of previous exacerbations, disease severity, poor disease-specific health status, gastroesophageal reflex, and non-private insurance status [8-11]. However, these studies were conducted within limited populations (e.g., patients in urban academic centers, and population in one state), thereby limiting generalizability of their results. Despite a substantial burden of COPD-related ED visits in an already stressed healthcare system, there have been no large cohort studies to characterize this high-risk patient population in the US.

To address these knowledge gaps, using large allpayer databases from two geographically dispersed states, we sought to quantify the proportion and characteristics of patients with frequent ED visits for AECOPD and associated healthcare burden, including rates of 30-day ED revisit, hospitalizations, near-fatal events, and hospital charges. A better understanding of these important issues in ED patients with AECOPD may inform potential strategies to improve their COPD management and reduce healthcare spending.

\section{Methods}

\section{Study design and setting}

We conducted a retrospective cohort study using data from the Healthcare Cost and Utilization Project (HCUP) State Emergency Department Databases (SEDD) and State Inpatient Databases (SID). The SEDD includes all treat-and-release and transfer ED visits from shortterm, acute-care, nonfederal, community hospitals in participating states. The SID includes all inpatient discharges from short-term, acute-care, nonfederal, general, and other specialty hospitals in participating states, including those admitted from the ED. Taken together, we identified all ED visits regardless of disposition and contained information on short-term outcomes for patients admitted through the ED. Additional details of the SEDD and SID can be found elsewhere $[12,13]$.

In this study, we used the data from California and Florida SEDD and SID in 2010 and 2011. These two states were selected for their geographic distribution, high data quality, and mainly because their databases included unique encrypted patient-level identifiers that enable follow-up of specific patients across years. The institutional review board of our hospital waived review of this study.

\section{Study population}

We identified all adults aged 40 years or older with at least one ED visit for AECOPD in 2010, by using International Classification of Diseases, Ninth Revision, Clinical Modification (ICD-9-CM) code for: 1) chronic bronchitis (491.xx), emphysema (492.xx), or chronic airway obstruction (496.xx) in the primary diagnosis field; or 2) acute respiratory failure (518.81, 518.82, or 518.84) listed in the primary diagnosis field and COPD listed as the secondary diagnosis (491.xx, 492.xx, or 496.xx). To minimize the potential misclassification of acute bronchitis as COPD, we did not include bronchitis not specified (490.xx). Likewise, we excluded patients younger than 40 years because they are less likely to have COPD [5]. We also excluded out-of-state residents, and patients who died at the first ED visit or hospitalization during the study period.

\section{Covariates}

The databases contain information on patient characteristics, including demographics (age, sex, and race/ ethnicity), primary insurance type, household income, urban-rural status, ICD-9-CM diagnosis, and patient comorbidities. The SEDD database also includes ED disposition. The patient characteristics at the first visit were used for the primary analysis. Primary insurance types were categorized into Medicaid, Medicare, private sources, self-pay, and other. Quartile classifications of estimated median household income of residents in the patient's ZIP Code were examined. These values were derived from the annual ZIP Code-demographic data and included in the SEDD and SID databases. Because these estimate are updated annually, the values ranges for the categories vary by year $[12,13]$. Urban-rural status of the patient residence was defined according to the National Center for Health Statistics [14]. To adjust for potential confounding by patient-mix, 29 Elixhauser comorbidity measures were derived based on the ICD9- $C M$ codes using the Agency for Healthcare Research and Quality (AHRQ) Comorbidity Software [15]. This 
risk adjustment tool has been derived and validated extensively [16].

\section{Outcome measures}

The primary outcome measure was the frequency of ED visits for AECOPD in a given year for each patient. The patient's first ED visit in 2010 was identified as the index ED visit. Each patient was then followed for 365 days after the index visit; then, the total number of ED visits for each patient was summated during the follow-up period, including the index visit.

Other outcome measures of interest were 30-day ED revisits, hospitalizations, near-fatal events, and charges for both ED and inpatient services. Thirty-day ED revisit was defined as an ED visit for AECOPD within 30 days of the previous ED or hospital discharge. Hospitalization was defined as a hospital admission for AECOPD from ED during the year after the index visit. Near-fatal event was defined as an ED visit or hospitalization for AECOPD involving noninvasive or invasive mechanical ventilation [17]; the use of mechanical ventilation was identified using the HCUP Clinical Classifications Software code 216. Charges reflect the total facility fees aggregated for a given individual; they are not available in the California datasets. All charges were converted to 2011 US dollars using the medical component of the Consumer Price Index [18].

\section{Statistical analysis}

For the purpose of this analysis, we categorized patients into three ED utilization groups according to the distribution of COPD-related ED visits and previous literature [8]: one ED visit (i.e., index visit only), two ED visits, and three or more ED visits within one year. First, we tested for unadjusted associations between patient-level variables and the frequency of ED visits for AECOPD using chi-square test or Kruskal-Wallis test, as appropriate. The patient-level covariates at the first visit were used for the analysis. Then, we fit multinomial logistic regression models to examine associations between patient-level variables and frequency of ED visits, with one ED visit group as the reference, adjusting for patient-mix using Elixhauser comorbidity measures.

Additionally, we calculated the rate of 30-day ED revisits, hospitalizations, and near-fatal events according to the ED visit utilization. The rates were defined as the total number of respective outcomes within a year of the index ED visit divided by the total number of ED visits for AECOPD. Then, we examined associations between the frequency of ED visits and these rates. We also performed linear regression at the patient-level to examine a linear association between the frequency of ED visits and charges.
In sensitivity analyses, to assess the consistency of associations between the frequency of ED visits and each outcome, we stratified the analysis by state, and included data from Nebraska. Data from Nebraska was not used for the primary analysis as they did not include race/ ethnicity. Additionally, to further investigate predictors of a higher frequency of ED visits, a negative binomial regression model with quasi-likelihood estimation was used [19]. This model has advantages that there is no need to define an arbitrary cutoff point and that this model appropriately accounts for statistical overdispersion [20,21]. All analyses were performed with SAS version 9.3 (SAS Institute, Cary, NC) and results were presented with $95 \%$ confidence interval (CI), when appropriate. A two-sided $\mathrm{P}$ value $<0.05$ was considered statistically significant.

\section{Results}

All ED visits for AECOPD made by patients aged 40 years or older in 2010 and $2011(n=294,678)$ were identified in the California and Florida databases. From this population, we sequentially excluded ED visits occurred $>365$ days after the index visit $(n=23,485)$, those made by patients who had no ED visits in 2010 $(\mathrm{n}=104,510)$, and those without a valid encrypted patient identifier $(n=6,583)$. We also excluded ED visits made by patients with out-of-state residence $(n=4,331)$ or who died at the index ED visit $(\mathrm{n}=1033)$. After these exclusions, the analytic cohort comprised 98,280 unique patients with 154,736 ED visits for AECOPD.

\section{ED visits for AECOPD}

Among this analytic cohort, 69,398 patients (70.6\%; 95\% CI, 70.3\%-70.9\%) reported one ED visit during the 1-year study period, while 28,882 patients (29.4\%; $95 \%$ CI, $29.1 \%$ $29.7 \%$ ) had two or more (frequent) visits. Figure 1 demonstrates the number of patients and ED visits for AECOPD during the study period by ED visit frequency. Patients with frequent visits accounted for 55.2\% (95\% CI, 54.9\%55.4\%) of all ED visits for AECOPD.

In the sensitivity analysis, the distribution of ED visit frequency was similar across California and Florida (Figure 2), with the comparable findings in Nebraska. For example, $29.7 \%$ of patients in California had frequent ED visits and accounted for 55.9\% (95\% CI, $55.5 \%-56,2 \%$ ) of all ED visits; $29.1 \%$ patients in Florida had frequent visits and accounted for $54.5 \%$ (95\% CI, 54.1\%-54.8\%) of all ED visits; $29.6 \%$ patients in Nebraska had frequent ED visits and accounted for 54.0\% (95\% CI, $52.5 \%-55.5 \%)$ of all ED visits.

\section{Patient characteristics}

Patient demographics, socioeconomic factors, and comorbidities differed across the ED visit frequency groups 


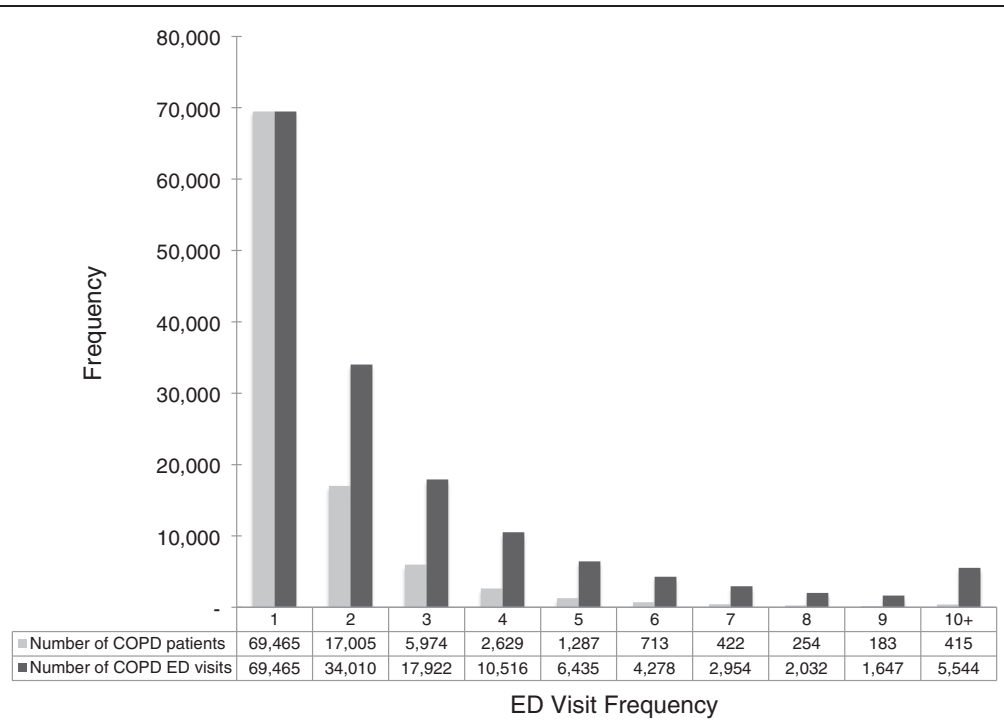

Figure 1 Number of patients and emergency department (ED) visits for acute exacerbation of chronic obstructive pulmonary disease (AECOPD), according to ED visit frequency.

(Table 1). Compared to patients with one ED visit, patients with a higher frequency of ED visits were more likely to be age 55-74 years, male sex, and non-Hispanic black race. Similarly, several markers of socioeconomic status, such as Medicaid insurance and lower median household income, were associated with a higher frequency of ED visits.

In a multinomial logistic regression model (Table 2), these patient characteristics remained significant as independent predictors of a higher frequency of ED visits across the frequency groups. For example, non-Hispanic white race (OR, 1.32; 95\% CI, 1.23-1.41),
non-Hispanic black race (OR, 1.59; 95\% CI, 1.45-1.73), Medicaid insurance (OR, 2.92; 95\% CI, 2.66-3.20), and lowest quartile for household income (OR, 1.29; 95\% CI, 1.21-1.38) were independently associated with three or more ED visits within one year. In addition, certain comorbidities such as congestive heart failure, pulmonary circulation disorders, depression, and psychoses were associated with a higher frequency of ED visits. In the sensitivity analyses, these associations remained statistically significant with stratification by state and the use of negative binomial regression model (Tables 3 and 4).

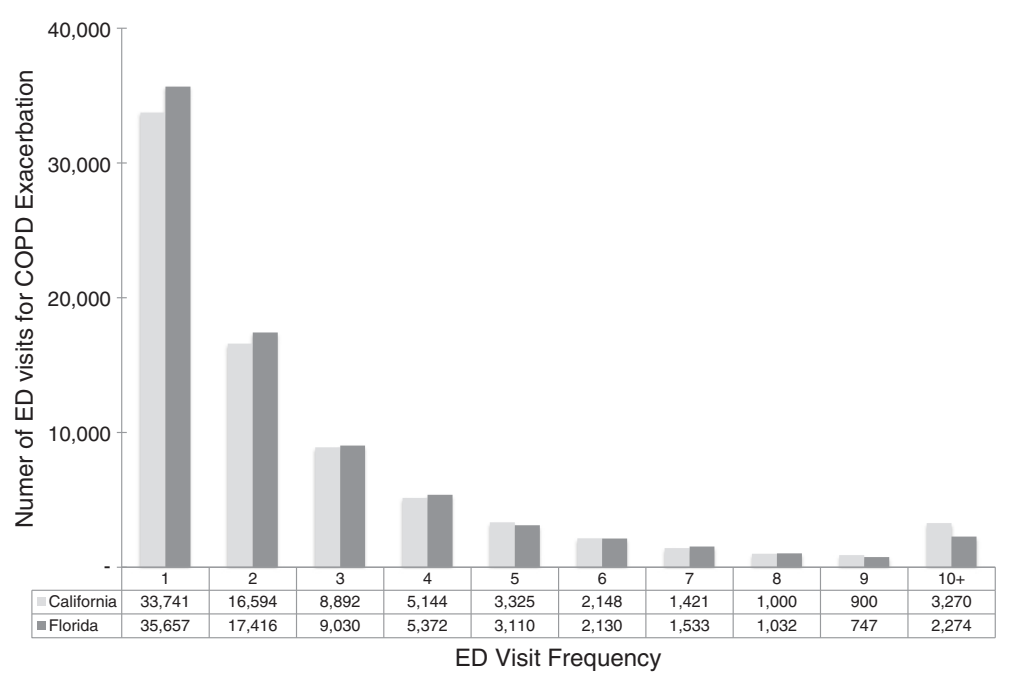

Figure 2 Number of emergency department (ED) visits for acute exacerbation of chronic obstructive pulmonary disease (AECOPD), according to ED visit frequency, stratified by state. 
Table 1 Demographic and Comorbidities of Patients with Chronic Obstructive Pulmonary Disease (COPD), according to the number of COPD-related emergency department visits in one year

\begin{tabular}{|c|c|c|c|c|}
\hline Variables* & $\begin{array}{c}1 \text { ED visit } \\
(n=69,398)\end{array}$ & $\begin{array}{l}2 \text { ED visits } \\
(n=17,005)\end{array}$ & $\begin{array}{c}\geq 3 \text { ED visits } \\
(n=11,877)\end{array}$ & P value $^{+}$ \\
\hline Age (y), median (IQR) & 69 (58-79) & $69(59-78)$ & $67(58-76)$ & $<0.001^{\ddagger}$ \\
\hline $40-54$ years & $11,922(17)$ & $2595(15)$ & $2010(17)$ & $<0.001$ \\
\hline $55-64$ years & $14,807(21)$ & $3815(22)$ & $3288(28)$ & \\
\hline $65-74$ years & $17,049(25)$ & $4575(27)$ & 3287 (28) & \\
\hline $75-84$ years & $16,788(24)$ & $4160(24)$ & $2441(21)$ & \\
\hline 85 years and older & $8832(13)$ & $1860(11)$ & $851(7)$ & \\
\hline Male sex & $30,633(44)$ & $7632(45)$ & $5527(47)$ & $<0.001$ \\
\hline Race/ethnicity & & & & $<0.001$ \\
\hline Non-hispanic white & $51,557(74)$ & $12,746(75)$ & $8713(73)$ & \\
\hline Non-hispanic black & $6349(9)$ & $1702(10)$ & $1577(13)$ & \\
\hline Hispanic & $7715(11)$ & $1732(10)$ & $1068(9)$ & \\
\hline Other & $2484(4)$ & $569(3)$ & $337(3)$ & \\
\hline Missing & $1303(2)$ & $256(2)$ & $182(2)$ & \\
\hline \multicolumn{5}{|l|}{ Insurance status } \\
\hline Medicare & $46,174(67)$ & $11,829(70)$ & $7793(66)$ & $<0.001$ \\
\hline Medicaid & $8092(12)$ & $2417(14)$ & $2413(20)$ & \\
\hline Private & $7931(11)$ & $1299(8)$ & $729(6)$ & \\
\hline Self-pay & $4322(6)$ & $71(4)$ & $431(4)$ & \\
\hline Other & $2870(4)$ & $699(4)$ & $508(4)$ & \\
\hline \multicolumn{4}{|c|}{ Quartiles for median household income of patient's ZIP code } & $<0.001$ \\
\hline 1 (lowest) & $22,390(32)$ & $5749(34)$ & $4415(37)$ & \\
\hline 2 & $19,307(28)$ & $4800(28)$ & $3325(28)$ & \\
\hline 3 & $15,904(23)$ & $3797(22)$ & $2497(21)$ & \\
\hline 4 (highest) & $10,524(15)$ & $2308(14)$ & $1372(12)$ & \\
\hline Missing & $1273(2)$ & $351(2)$ & $268(2)$ & \\
\hline Patient residence & & & & $<0.001$ \\
\hline Counties of $\geq 1$ million population & $42,303(61)$ & $10,245(60)$ & $7123(60)$ & \\
\hline Counties of $250,000-999,999$ population & $17,379(25)$ & $4319(25)$ & $2983(25)$ & \\
\hline Counties of 50,000-249,999 population & $4103(6)$ & $1053(6)$ & $750(6)$ & \\
\hline Counties of $<50,000$ population & $5590(8)$ & $1335(8)$ & $956(8)$ & \\
\hline State & & & & 0.02 \\
\hline California & $33,741(49)$ & $8297(49)$ & $5942(50)$ & \\
\hline Florida & $35,657(51)$ & $8708(51)$ & $5935(50)$ & \\
\hline Any comorbidities & $54,633(79)$ & $13,888(82)$ & $9644(81)$ & $<0.001$ \\
\hline \multicolumn{5}{|l|}{ Selected comorbidities ${ }^{\S}$} \\
\hline Congestive heart failure & $13,607(20)$ & $3831(23)$ & $2708(23)$ & $<0.001$ \\
\hline Pulmonary circulation disorders & $674(1)$ & $269(2)$ & $198(2)$ & $<0.001$ \\
\hline Diabetes, uncomplicated & $14,813(21)$ & $3931(23)$ & $2939(25)$ & $<0.001$ \\
\hline Obesity & $7195(10)$ & $1935(11)$ & $1437(12)$ & $<0.001$ \\
\hline Depression & $6357(9)$ & $1886(11)$ & $1479(12)$ & $<0.001$ \\
\hline Psychoses & $3233(5)$ & $1001(6)$ & $847(7)$ & $<0.001$ \\
\hline Drug abuse & $1430(2)$ & $458(3)$ & $528(4)$ & $<0.001$ \\
\hline
\end{tabular}

Abbreviations: COPD chronic obstructive pulmonary disease, $E D$ emergency department, IQR interquartile range.

*Data were expressed as $\mathrm{n}(\%)$ unless otherwise indicated.

${ }^{+}$Unadjusted comparisons were tested using chi-square test unless otherwise indicated.

‡Unadjusted comparison was tested using Kruskal-Wallis test.

${ }^{\S}$ Comorbidity was defined as at least 1 Elixhauser comorbidity measure. 
Table 2 Multinomial regression models for factors associated with frequent emergency department visits for acute exacerbation of chronic obstructive pulmonary disease

\begin{tabular}{|c|c|c|c|c|}
\hline \multirow[b]{2}{*}{ Variables } & \multicolumn{2}{|c|}{2 (vs. 1) ED visits } & \multicolumn{2}{|c|}{$\geq 3$ (vs. 1) ED visits } \\
\hline & Odds ratio $(95 \% \mathrm{Cl})$ & $P$ value & Odds ratio $(95 \% \mathrm{Cl})$ & $P$ value \\
\hline Age, y & & $<0.001$ & & $<0.001$ \\
\hline 40-54 years & 1 [reference] & & 1 [reference] & \\
\hline $55-64$ years & $1.15(1.09-1.22)$ & $<0.001$ & $1.31(1.23-1.39)$ & $<0.001$ \\
\hline $65-74$ years & $1.14(1.07-1.22)$ & $<0.001$ & $1.11(1.03-1.20)$ & 0.006 \\
\hline $75-84$ years & $1.05(0.98-1.12)$ & 0.10 & $0.86(0.79-0.93)$ & $<0.001$ \\
\hline 85 years and older & $0.90(0.83-0.97)$ & 0.03 & $0.58(0.52-0.64)$ & $<0.001$ \\
\hline Male sex & $1.04(1.00-1.08)$ & 0.048 & $1.12(1.07-1.17)$ & $<0.001$ \\
\hline \multicolumn{5}{|l|}{ Race/ethnicity } \\
\hline Non-hispanic white & $1.15(1.08-1.21)$ & $<0.001$ & $1.32(1.23-1.41)$ & $<0.001$ \\
\hline Non-hispanic black & $1.17(1.09-1.27)$ & $<0.001$ & $1.59(1.46-1.73)$ & $<0.001$ \\
\hline Hispanic & 1 [reference] & & 1 [reference] & \\
\hline Other & $1.07(0.96-1.19)$ & 0.29 & $1.07(0.96-1.19)$ & 0.33 \\
\hline \multicolumn{5}{|l|}{ Insurance status } \\
\hline Medicare & $1.58(1.47-1.69)$ & $<0.001$ & $2.10(1.92-2.29)$ & $<0.001$ \\
\hline Medicaid & $1.77(1.64-1.92)$ & $<0.001$ & $2.92(2.66-3.20)$ & $<0.001$ \\
\hline Private & 1 [reference] & & 1 [reference] & \\
\hline Self-pay & $1.11(1.00-1.23)$ & 0.046 & $1.05(0.92-1.20)$ & 0.45 \\
\hline Other & $1.46(1.32-1.62)$ & $<0.001$ & $1.78(1.57-2.02)$ & $<0.001$ \\
\hline \multicolumn{5}{|c|}{ Quartiles for median household income of patient's ZIP code } \\
\hline 1 (lowest) & $1.13(1.07-1.20)$ & $<0.001$ & $1.29(1.21-1.38)$ & $<0.001$ \\
\hline 2 & $1.11(1.05-1.18)$ & $<0.001$ & $1.22(1.14-1.31)$ & $<0.001$ \\
\hline 3 & $1.08(1.01-1.14)$ & 0.01 & $1.14(1.06-1.22)$ & $<0.001$ \\
\hline 4 (highest) & 1 [reference] & & 1 [reference] & \\
\hline \multicolumn{5}{|l|}{ Patient residence } \\
\hline Counties of $\geq 1$ million population & $1.07(1.00-1.15)$ & 0.06 & $1.09(1.01-1.18)$ & 0.03 \\
\hline Counties of 250,000-999,999 population & $1.07(1.00-1.15)$ & 0.07 & $1.09(1.00-1.18)$ & 0.06 \\
\hline Counties of $50,000-249,999$ population & $1.09(1.00-1.20)$ & 0.06 & $1.10(0.99-1.23)$ & 0.09 \\
\hline Counties of $<50,000$ population & 1 [reference] & & 1 [reference] & \\
\hline \multicolumn{5}{|l|}{ State } \\
\hline California & $1.01(0.98-1.05)$ & 0.44 & $1.06(1.02-1.11)$ & 0.006 \\
\hline Florida & 1 [reference] & & 1 [reference] & \\
\hline \multicolumn{5}{|l|}{ Selected comorbidities* } \\
\hline Congestive heart failure & $1.17(1.11-1.22)$ & $<0.001$ & $1.24(1.17-1.30)$ & $<0.001$ \\
\hline Pulmonary circulation disorders & $1.41(1.22-1.64)$ & $<0.001$ & $1.39(1.17-1.64)$ & $<0.001$ \\
\hline Diabetes, uncomplicated & $1.05(1.01-1.10)$ & 0.02 & $1.13(1.07-1.19)$ & $<0.001$ \\
\hline Obesity & $0.99(0.94-1.05)$ & 0.79 & $0.97(0.91-1.03)$ & 0.31 \\
\hline Depression & $1.21(1.14-1.28)$ & $<0.001$ & $1.38(1.30-1.47)$ & $<0.001$ \\
\hline Psychoses & $1.17(1.09-1.27)$ & $<0.001$ & $1.27(1.17-1.38)$ & $<0.001$ \\
\hline Drug abuse & $1.22(1.09-1.37)$ & $<0.001$ & $1.66(1.48-1.85)$ & $<0.001$ \\
\hline
\end{tabular}

Abbreviations: COPD chronic obstructive pulmonary disease, $E D$ emergency department, $\mathrm{Cl}$ confidence interval. Bold results are statistically significant.

*Non-significant Elixhauser comorbidity measures across the frequency groups included peripheral vascular disease, hypertension, neurological disorders, diabetes with complications, liver disease, peptic ulcer disease, acquired immunodeficiency syndrome, lymphoma, solid tumor without metastasis, coagulopathy, fluid and electrolyte disorders, anemia, and alcohol abuse. 
Table 3 Multinomial regression models for factors associated with frequent emergency department visits for acute exacerbation of chronic obstructive pulmonary disease, stratified by state

\begin{tabular}{|c|c|c|c|c|c|c|c|c|}
\hline \multirow[b]{3}{*}{ Variables } & \multicolumn{4}{|c|}{ California } & \multicolumn{4}{|c|}{ Florida } \\
\hline & \multicolumn{2}{|c|}{2 (vs. 1) ED visits } & \multicolumn{2}{|c|}{$\geq 3$ (vs. 1) ED visits } & \multicolumn{2}{|c|}{2 (vs. 1) ED visits } & \multicolumn{2}{|c|}{$\geq 3$ (vs. 1) ED visits } \\
\hline & $\begin{array}{l}\text { Odds ratio } \\
(95 \% \mathrm{Cl})\end{array}$ & $\begin{array}{c}P \\
\text { value }\end{array}$ & $\begin{array}{l}\text { Odds ratio } \\
(95 \% \mathrm{Cl})\end{array}$ & $\begin{array}{c}P \\
\text { value }\end{array}$ & $\begin{array}{l}\text { Odds ratio } \\
(95 \% \mathrm{Cl})\end{array}$ & $\begin{array}{c}P \\
\text { value }\end{array}$ & $\begin{array}{l}\text { Odds ratio } \\
(95 \% \mathrm{Cl})\end{array}$ & $\begin{array}{c}P \\
\text { value }\end{array}$ \\
\hline \multicolumn{9}{|l|}{ Age, y } \\
\hline $40-54$ years & 1 [reference] & & 1 [reference] & & 1 [reference] & & 1 [reference] & \\
\hline $55-64$ years & $1.20(1.11-1.31)$ & $<0.001$ & $1.32(1.21-1.45)$ & $<0.001$ & $1.11(1.03-1.21)$ & 0.008 & $1.28(1.17-1.41)$ & $<0.001$ \\
\hline $65-74$ years & $1.17(1.07-1.29)$ & 0.001 & $1.16(1.04-1.29)$ & 0.008 & $1.13(1.03-1.23)$ & 0.01 & $1.05(0.95-1.17)$ & 0.34 \\
\hline $75-84$ years & $1.15(1.04-1.27)$ & 0.007 & $0.90(0.80-1.01)$ & 0.08 & $0.98(0.89-1.08)$ & 0.73 & $1.81(0.73-0.91)$ & $<0.001$ \\
\hline 85 years and older & $0.99(0.88-1.11)$ & 0.83 & $0.61(0.53-0.70)$ & $<0.001$ & $0.85(0.76-0.96)$ & 0.007 & $0.54(0.47-0.63)$ & $<0.001$ \\
\hline Male sex & $1.05(1.00-1.10)$ & 0.048 & $1.13(1.06-1.19)$ & $<0.001$ & $1.03(0.98-1.08)$ & 0.30 & $1.11(1.05-1.18)$ & $<0.001$ \\
\hline \multicolumn{9}{|l|}{ Race/ethnicity } \\
\hline Non-hispanic white & $1.15(1.06-1.25)$ & 0.001 & $1.32(1.20-1.46)$ & $<0.001$ & $1.13(1.03-1.23)$ & 0.004 & $1.30(1.17-1.44)$ & $<0.001$ \\
\hline Non-hispanic black & $1.24(1.11-1.38)$ & 0.001 & $1.71(1.51-1.93)$ & $<0.001$ & $1.12(1.01-1.25)$ & 0.04 & $1.49(1.31-1.69)$ & $<0.001$ \\
\hline Hispanic & 1 [reference] & & 1 [reference] & & 1 [reference] & & 1 [reference] & \\
\hline Other & $1.23(1.00-1.28)$ & 0.06 & $1.16(0.99-1.35)$ & 0.06 & $0.85(0.65-1.10)$ & 0.21 & $0.80(0.57-1.12)$ & 0.20 \\
\hline \multicolumn{9}{|l|}{ Insurance status } \\
\hline Medicare & $1.55(1.41-1.71)$ & $<0.001$ & $1.88(1.67-2.11)$ & $<0.001$ & $1.63(1.47-1.81)$ & $<0.001$ & $2.41(2.11-2.75)$ & $<0.001$ \\
\hline Medicaid & $1.65(1.48-1.83)$ & $<0.001$ & $2.58(2.29-2.92)$ & $<0.001$ & $1.96(1.75-2.20)$ & $<0.001$ & $3.38(2.93-3.89)$ & $<0.001$ \\
\hline Private & 1 [reference] & & 1 [reference] & & 1 [reference] & & 1 [reference] & \\
\hline Self-pay & $1.10(0.95-1.28)$ & 0.21 & $1.05(0.87-1.27)$ & 0.62 & $1.13(0.98-1.29)$ & 0.09 & $1.11(0.92-1.33)$ & 0.29 \\
\hline Other & $1.34(1.14-1.57)$ & $<0.001$ & $1.34(1.10-1.62)$ & 0.004 & $1.58(1.37-1.81)$ & $<0.001$ & $2.25(1.90-2.67)$ & $<0.001$ \\
\hline \multicolumn{9}{|l|}{$\begin{array}{l}\text { Quartiles for median household } \\
\text { income of patient's ZIP code }\end{array}$} \\
\hline 1 (lowest) & $1.24(1.13-1.37)$ & $<0.001$ & $1.18(1.09-1.28)$ & $<0.001$ & $1.10(1.01-1.19)$ & 0.03 & $1.22(1.11-1.34)$ & $<0.001$ \\
\hline 2 & $1.22(1.12-1.32)$ & $<0.001$ & $1.24(1.13-1.37)$ & $<0.001$ & $1.03(0.95-1.11)$ & 0.49 & $1.19(1.08-1.31)$ & $<0.001$ \\
\hline 3 & $1.12(1.03-1.21)$ & 0.001 & $1.20(1.09-1.33)$ & $<0.001$ & $1.04(0.95-1.13)$ & 0.40 & $1.07(0.97-1.19)$ & 0.19 \\
\hline 4 (highest) & 1 [reference] & & 1 [reference] & & 1 [reference] & & 1 [reference] & \\
\hline \multicolumn{9}{|l|}{ Patient residence } \\
\hline Counties of $\geq 1$ million population & $1.00(0.89-1.12)$ & 0.99 & $0.97(0.86-1.11)$ & 0.69 & $1.10(1.01-1.20)$ & 0.03 & $1.16(1.04-1.28)$ & 0.005 \\
\hline $\begin{array}{l}\text { Counties of 250,000-999,999 } \\
\text { population }\end{array}$ & $1.06(0.94-1.20)$ & 0.34 & $1.02(0.89-1.17)$ & 0.78 & $1.07(0.98-1.17)$ & 0.16 & $1.09(0.98-1.22)$ & 0.10 \\
\hline $\begin{array}{l}\text { Counties of 50,000-249,999 } \\
\text { population }\end{array}$ & $1.01(0.87-1.06)$ & 0.92 & $1.04(0.89-1.22)$ & 0.65 & $1.16(1.02-1.32)$ & 0.02 & $1.10(0.94-1.28)$ & 0.23 \\
\hline Counties of $<50,000$ population & 1 [reference] & & 1 [reference] & & 1 [reference] & & 1 [reference] & \\
\hline \multicolumn{9}{|l|}{ Selected comorbidities } \\
\hline Congestive heart failure & $1.16(1.09-1.23)$ & $<0.001$ & $1.27(1.18-1.36)$ & $<0.001$ & $1.18(1.11-1.26)$ & $<0.001$ & $1.22(1.13-1.31)$ & $<0.001$ \\
\hline Pulmonary circulation disorders & $1.51(1.22-1.86)$ & $<0.001$ & $1.34(1.04-1.73)$ & 0.02 & $1.29(1.05-1.60)$ & 0.02 & $1.37(1.09-1.73)$ & 0.008 \\
\hline Diabetes, uncomplicated & $1.02(0.96-1.09)$ & 0.47 & $1.14(1.06-1.23)$ & $<0.001$ & $1.08(1.02-1.15)$ & 0.009 & $1.12(1.05-1.20)$ & $<0.001$ \\
\hline Obesity & $1.01(0.92-1.10)$ & 0.85 & $0.96(0.87-1.06)$ & 0.42 & $0.98(0.91-1.06)$ & 0.60 & $0.97(0.89-1.06)$ & 0.50 \\
\hline Depression & $1.22(1.11-1.33)$ & $<0.001$ & $1.39(1.26-1.54)$ & $<0.001$ & $1.19(1.10-1.28)$ & $<0.001$ & $1.37(1.26-1.49)$ & $<0.001$ \\
\hline Psychoses & 1.19 (1.07-1.32) & 0.001 & $1.26(1.13-1.42)$ & $<0.001$ & $1.16(1.04-1.29)$ & 0.01 & $1.28(1.14-1.45)$ & $<0.001$ \\
\hline Drug abuse & $1.27(1.10-1.47)$ & 0.001 & $1.72(1.49-1.97)$ & $<0.001$ & $1.16(0.97-1.39)$ & 0.10 & $1.55(1.30-1.86)$ & $<0.001$ \\
\hline
\end{tabular}

Abbreviations: COPD chronic obstructive pulmonary disease, $E D$ emergency department, $C l$ confidence interval. Bold results are statistically significant. 
Table 4 Negative binomial regression model for factors associated with frequency of emergency department visits for acute exacerbation of chronic obstructive pulmonary disease

\begin{tabular}{|c|c|c|}
\hline Variables & Risk ratio $(95 \% \mathrm{Cl})$ & $P$ value \\
\hline \multicolumn{3}{|l|}{ Age, y } \\
\hline $40-54$ years & 1 [reference] & \\
\hline $55-64$ years & $1.07(1.06-1.09)$ & $<0.001$ \\
\hline $65-74$ years & $1.02(1.00-1.03)$ & 0.04 \\
\hline $75-84$ years & $0.94(0.92-0.96)$ & $<0.001$ \\
\hline 85 years and older & $0.86(0.85-0.88)$ & $<0.001$ \\
\hline \multicolumn{3}{|l|}{ Male sex } \\
\hline \multicolumn{3}{|l|}{ Race/ethnicity } \\
\hline Non-hispanic white & $1.08(1.06-1.09)$ & $<0.001$ \\
\hline Non-hispanic black & $1.15(1.13-1.17)$ & $<0.001$ \\
\hline Hispanic & 1 [reference] & \\
\hline Other & $1.01(0.98-1.03)$ & 0.62 \\
\hline \multicolumn{3}{|l|}{ Insurance status } \\
\hline Medicare & $1.21(1.19-1.23)$ & $<0.001$ \\
\hline Medicaid & $1.34(1.32-1.36)$ & $<0.001$ \\
\hline Private & 1 [reference] & \\
\hline Self-pay & $1.01(0.99-1.03)$ & 0.45 \\
\hline Other & $1.13(1.10-1.16)$ & $<0.001$ \\
\hline \multicolumn{3}{|c|}{ Quartiles for median household income of patient's ZIP code } \\
\hline 1 (lowest) & $1.06(1.05-1.08)$ & $<0.001$ \\
\hline 2 & $1.05(1.03-1.06)$ & $<0.001$ \\
\hline 3 & $1.03(1.01-1.04)$ & $<0.001$ \\
\hline 4 (highest) & 1 [reference] & \\
\hline \multicolumn{3}{|l|}{ Patient residence } \\
\hline Counties of $\geq 1$ million population & $1.03(1.01-1.05)$ & $<0.001$ \\
\hline Counties of 250,000-999,999 population & $1.04(1.02-1.05)$ & $<0.001$ \\
\hline Counties of 50,000-249,999 population & $1.03(1.01-1.06)$ & 0.007 \\
\hline Counties of $<50,000$ population & 1 [reference] & \\
\hline \multicolumn{3}{|l|}{ State } \\
\hline California & $1.02(1.02-1.03)$ & $<0.001$ \\
\hline Florida & 1 [reference] & \\
\hline \multicolumn{3}{|l|}{ Selected comorbidities } \\
\hline Congestive heart failure & $1.05(1.04-1.06)$ & $<0.001$ \\
\hline Pulmonary circulation disorders & $1.13(1.09-1.17)$ & $<0.001$ \\
\hline Diabetes, uncomplicated & $1.04(1.03-1.05)$ & $<0.001$ \\
\hline Obesity & $0.97(0.96-0.99)$ & $<0.001$ \\
\hline Depression & $1.08(1.07-1.10)$ & $<0.001$ \\
\hline Psychoses & $1.08(1.06-1.10)$ & $<0.001$ \\
\hline Drug abuse & $1.18(1.15-1.21)$ & $<0.001$ \\
\hline
\end{tabular}

Abbreviations: COPD chronic obstructive pulmonary disease, ED emergency department $\mathrm{Cl}$ confidence interval. Bold results are statistically significant. 
Table 5 Outcomes of patients with Chronic Obstructive Pulmonary Disease (COPD), according to the number of COPDrelated emergency department visits in one year

\begin{tabular}{|c|c|c|c|c|}
\hline Variables & 1 ED visit & 2 ED visits & $\geq 3$ ED visits & $P$ value \\
\hline Number of ED visits for COPD, no. & 69,398 & 34,010 & 51,328 & - \\
\hline 30-day ED revisit rate for COPD, \% $(95 \% \mathrm{Cl})^{*}$ & - & $10.5(10.1-10.8)$ & $30.0(29.6-30.4)$ & $<0.001$ \\
\hline Hospitalization rate for COPD, \% (95\% Cl) & $62.3(62.0-62.7)$ & $64.9(64.4-65.4)$ & $62.1(61.6-62.5)$ & $<0.001$ \\
\hline Near-fatal event rate, \% (95\% Cl) & $5.8(5.6-6.0)$ & $8.2(7.9-8.5)$ & $8.8(8.6-9.1)$ & $<0.001$ \\
\hline
\end{tabular}

Abbreviations: COPD chronic obstructive pulmonary disease $E D$ emergency department, $I Q R$ interquartile range, $C l$ confidence interval.

*Excluding the index ED visits and hospitalizations.

\section{COPD 30-day ED revisits, hospitalizations, and near-fatal} events

Table 5 summarizes clinical outcomes at the visit-level. Overall, $12.3 \%$ (95\% CI, 12.1\%-12.4\%) of ED visits for AECOPD were 30-day revisit events. Patients with a higher frequency of ED visits were more likely to develop a 30-day ED revisit (Table 5), regardless of state (all $\mathrm{P}<0.001$; Table 6).

Overall, approximately two-thirds of ED visits $(62.8 \%$; 95\% CI, 62.6\%-63.0\%) resulted in a hospitalization. Hospitalization rates were greater than $60 \%$ across the ED frequency groups and highest with the patients with two ED visits (Table 5). Patients with frequent ED visits accounted for 55.5\% (95\% CI, 55.2\%-55.8\%) of total hospitalizations for AECOPD.

Additionally, 7.3\% (95\% CI, 7.2\%-7.5\%) of ED visits led to a near-fatal event. Patients with a higher frequency of ED visits had a higher chance of near-fatal event during the 1 -year period, regardless of state (all $\mathrm{P}<0.001$; Tables 5 and 6). Patients with frequent ED visits accounted for $64.4 \%$ (95\% CI, 63.5\%-65.3\%) of near-fatal events.

\section{Hospital charges for AECOPD in Florida}

The total charges for ED and inpatient services for AECOPD were \$1.94 billion (95\% CI, \$1.90-1.97 billion) in Florida. Patients with frequent ED visits accounted for the majority of total charges ( $\$ 1.07$ billion; 95\% CI, \$1.04-1.09 billion); this was driven by a significant linear association between number of ED visits and charges per patient $(\mathrm{P}<0.001$; Figure 3$)$. In addition, recurrent visits after the index ED visits accounted for $37.1 \%$ of total charges (\$719 million; 95\% CI, \$709-731 million).

\section{Discussion}

Our study of 98,280 COPD patients showed that 29\% of the patients had frequent ED visits for AECOPD (2 or more ED visits in a year). The societal burden of this population with COPD - as measured by ED visits, hospitalizations, and near-fatal events - was large. Indeed, this high-risk population accounted for $55 \%$ of all ED visits, 56\% of all COPD hospitalizations, $64 \%$ of near-fatal events, all contributing to substantial healthcare spending. We also found that male sex, non-Hispanic

Table 6 Outcomes of chronic obstructive pulmonary disease patients, according to the number of emergency department visits, stratified by state

\begin{tabular}{|c|c|c|c|c|}
\hline Variables & 1 ED visit & 2 ED visits & $\geq 3$ ED visits & $P$ value \\
\hline \multicolumn{5}{|l|}{ California } \\
\hline Number of ED visits for COPD, no. & 33,741 & 16,594 & 26,100 & - \\
\hline 30-day ED revisit rate for COPD, \% $(95 \% \mathrm{Cl})^{*}$ & - & $11.1(10.6-11.6)$ & $31.7(31.2-32.3)$ & $<0.001$ \\
\hline Hospitalization rate for COPD, \% (95\% Cl) & $55.7(55.2-56.2)$ & $54.6(53.9-55.4)$ & $54.5(53.9-55.1)$ & $<0.001$ \\
\hline Near-fatal event rate, \% (95\% Cl) & $6.8(6.5-7.1)$ & $9.2(8.7-9.6)$ & $9.6(9.2-9.9)$ & $<0.001$ \\
\hline \multicolumn{5}{|l|}{ Florida } \\
\hline Number of ED visits for COPD, no. & 35,657 & 17,416 & 25,228 & - \\
\hline 30-day ED revisit rate for COPD, \% $(95 \% \mathrm{Cl})^{*}$ & - & $9.8(9.4-10.3)$ & $28.2(27.6-28.8)$ & $<0.001$ \\
\hline Hospitalization rate for COPD, \% (95\% Cl) & $68.6(68.1-69.0)$ & $71.7(71.0-72.3)$ & $69.9(69.3-70.4)$ & $<0.001$ \\
\hline Near-fatal event rate, \% (95\% Cl) & $4.9(4.7-5.1)$ & $7.3(6.9-7.7)$ & $9.9(9.5-10.3)$ & $<0.001$ \\
\hline \multicolumn{5}{|l|}{ Nebraska } \\
\hline Number of ED visits for COPD, no. & 2009 & 1060 & 1301 & \\
\hline 30-day ED revisit rate for COPD, \% $(95 \% \mathrm{Cl})^{*}$ & - & $8.6(7.0-10.4)$ & $27.2(24.8-29.7)$ & $<0.001$ \\
\hline Hospitalization rate for COPD, \% (95\% Cl) & $52.5(50.3-54.7)$ & $56.6(53.6-59.6)$ & $53.0(50.2-55.7)$ & 0.08 \\
\hline Near-fatal event rate, \% (95\% Cl) & $6.9(5.8-8.1)$ & $7.9(6.4-9.7)$ & $11.2(9.6-13.1)$ & $<0.001$ \\
\hline
\end{tabular}

Abbreviations: COPD chronic obstructive pulmonary disease, $E D$ emergency department, IQR interquartile range $C l$ confidence interval. *Excluding the index ED visits and hospitalizations. 


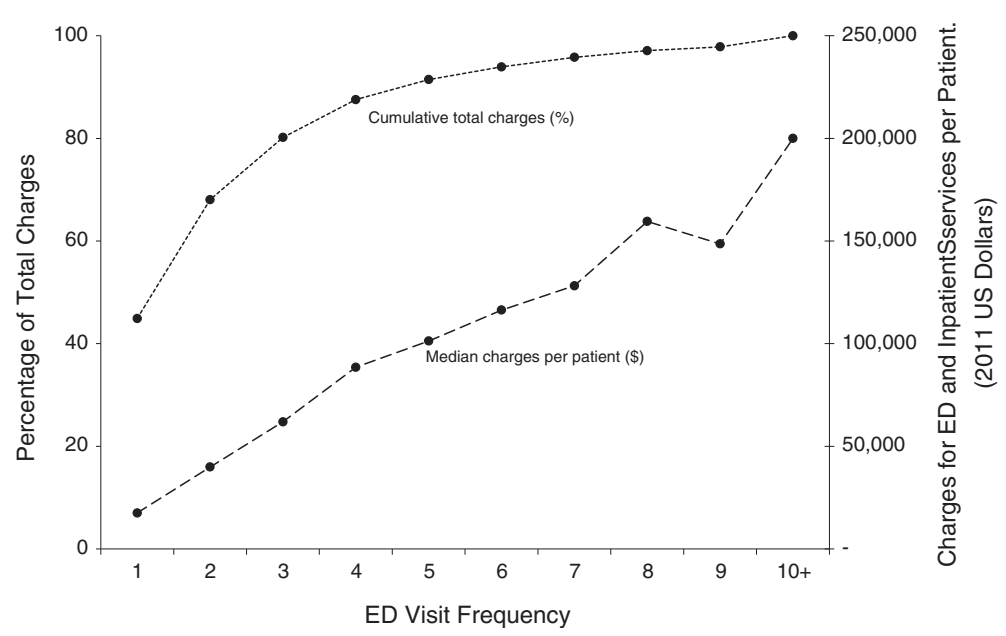

Figure 3 Median and cumulative charges for emergency department (ED) and inpatient services for acute exacerbation of chronic obstructive pulmonary disease (AECOPD), according to ED visit frequency. There was a significant linear association between frequency of ED visits and charges per patient $(P<0.001)$.

white and black race, and lower socioeconomic status were significantly associated with a higher frequency of ED visits. These findings inform potential strategies to reduce the large public health burden from AECOPD.

\section{Proportion of frequent ED visits for AECOPD}

To date, most clinical research on patients with AECOPD has focused on predicting and preventing acute relapse and 30-day readmission [22-24]. However, the predictors of acute relapse are not necessarily the predictors of frequent ED visits over longer periods. Conceptually, relapse is more likely to be associated with acute or medical factors, such as severity of exacerbation and quality of care at the index ED visit or hospitalization [24]. In contrast to acute relapse, recurrent ED visits for AECOPD over the course of a longer period of time, such as 1 year in our study, have a broader meaning because it may reflect a failure of less costly and more prevention-oriented COPD care.

A multicenter ED-based study in the early 2000s found that $64 \%$ of ED patients with AECOPD selfreported frequent ED visits within past year [11]. By contrast, a claims data study from North Carolina found that $28 \%$ of ED patients with AECOPD had frequent ED visits in the late 2000s [10]. Between 2010 and 2011, we demonstrated that $29 \%$ of ED patients with AECOPD had frequent ED visits in the two large, geographicallydispersed states, with a comparable percentage in a third state.

The reasons for the differences in the proportion of frequent ED visits for AECOPD among the studies are unclear and likely multifactorial. The population of the earlier multicenter study was limited to urban teaching hospitals that might have disproportionately high COPD morbidity, and thereby led to more frequent ED visits. Alternatively, the observed lower proportion of frequent ED visits might reflect declines in the rate of severe AECOPD over the past decade $[1,25]$. Secular changes in multiple factors might have contributed to such a potential improvement, including favorable changes in personal health behavior (e.g., smoking), self-management education, access to longitudinal care, hospital-at-home care, and dissemination of evidencebased therapy. For example, the release of Global Initiatives for Chronic Obstructive Lung Disease guidelines in 2001, 2006, and 2011 may have helped reduce the rate of AECOPD [26]. Indeed, after the publication of the first guidelines, researchers reported increases in the use of long-acting beta-agonists with corticosteroids [27] and influenza vaccinations [28] as well as reductions in smoking rate [29]. However, although COPD care has improved, the use of recommended medication combinations and stage-appropriate treatment still has ample room for improvement [30]. The observed lower proportion of frequent ED visits for AECOPD in 20102011 supports cautious optimism that COPD morbidity can be prevented and the societal burden reduced. Yet, considering the previous knowledge that only half of patients with AECOPD seek care in the ED or outpatient clinics [31], the large remaining burden underscores the need for continued secondary prevention efforts among patients with COPD.

\section{Characteristics of patients with frequent ED visits for AECOPD}

We were also struck by the disproportionate socioeconomic disparity in frequency of ED visits for AECOPD. In the present study, we found that adults at highest 
risk of frequent ED visits were more likely to have public insurance, and to have lower socioeconomic status. These results support prior reports of higher rates of frequent ED visits for COPD among these vulnerable populations $[10,11]$. Although the precise role of lower socioeconomic status to this disparity in COPD healthcare utilizations is unclear [5], the asthma literature would suggest that differences in health beliefs, less self-management education, and limited access to preventive and specialist care in this population might lead to a heavier reliance on episodic symptom treatment and emergency care [32]. Other potential factors that might explain the difference in ED use across socioeconomic status include degree of past and current smoking, living conditions, outpatient treatment, and COPD-specific disease severity [5,33]. The pathway through which socioeconomic status affects healthcare utilization is undoubtedly complex; further investigation is warranted to better understand the underlying reasons for this disparity in ED utilization among patients with AECOPD.

\section{Burden of patients with frequent ED visits for AECOPD}

Consistent with the previous study from North Carolina [10], we observed substantial patient morbidity, as measured by ED visits and hospitalizations for AECOPD. Additionally, our study extended prior research by documenting relatively high use of intensive care resources and high healthcare spending in frequent ED users with AECOPD. Indeed, patients with frequent ED visits accounted for only $29 \%$ of all patients; however, they comprised $64 \%$ of mechanical ventilation use and $55 \%$ of the total charges for ED and inpatient services.

COPD is designated as an ambulatory care sensitive condition by the AHRQ, among other conditions [34]; ED visits for AECOPD can often be prevented by evidencebased outpatient care (e.g., smoking cessation, influenza and pneumococcal vaccines, knowledge of current therapy including inhaler technique, and evidence-based pharmacologic treatment) [5]. Therefore, organized efforts to develop and implement systems of care are imperative to lower COPD adverse outcomes and healthcare expenditures. If recurrent ED visits could be prevented in this study, there would have been approximately 56,456 prevented $E D$ visits and 35,520 prevented hospitalizations in these two states alone. In terms of cost, this would have saved 719 million dollars in Florida alone.

Although these administrative data are unable to explore more granular aspects of COPD care, our findings can better inform a system of care for patients with AECOPD. At the individual patient level, the use of patient characteristics to identify patients at high-risk for frequent AECOPD remains limited [35]. The present findings underscore the importance of translating high-quality research into the risk stratification, coupled with dissemination of these finding to improve care for patients with COPD. At the health system level, our observation should facilitate further work on how to reduce the enormous and uneven public heath burden by targeting the population at greatest risk for integrated research, health policy, and community action.

\section{Potential limitations}

Our study should be viewed in the context of several potential limitations. Our data were not derived from a sample of the national COPD population. However, the data from two geographically diverse states included all ED visits and hospitalizations for approximately $19 \%$ of the US population [36]; the age and sex distributions of the ED visits mirrored a national surveillance of COPDrelated ED visits [1]. Moreover, our findings persisted across the two geographically dispersed states, with the comparable findings in a third state, suggesting a potential generalizability at the nation-level. Secondly, as with any studies using administrative data, there may be some misclassification of medical claims. However, HCUP data are highly accurate, rigorously tested, and widely used to estimate diagnoses and visit frequency $[37,38]$. Thirdly, our data do not include information on outpatient treatment and COPD-specific disease severity measures. As a surrogate for severity for COPD, we controlled for Elixhauser comorbidity measures in our analysis. In addition, our study did not examine care coordination, such as early discharge to pulmonary rehabilitation, or nurse or self-management education $[39,40]$. Fourthly, some of the unadjusted comparisons of patient characteristics were statistically significant with a marginal clinical significance because of the large numbers. Lastly, our objective was to assess COPD-related ED visits and associated healthcare utilization. Some patients may have had visits to urgent care or other ambulatory care sites; this would have led to an underestimation of healthcare utilization for AECOPD. However, because we focused on the characteristics and burden of the frequent ED utilizers for COPD, our observations are of direct relevance to the development of strategies to improve COPD care in this important patient population.

\section{Conclusions}

By using large all-payer databases from two geographically dispersed states in US, we found that $29 \%$ of the ED patients with AECOPD had frequent ED visits and that lower socioeconomic status were significantly associated with a higher frequency of ED visits. This population accounts for the majority of all COPD-related ED visits, hospitalizations, near-fatal events, and hospital charges, and present an important public health challenge. Our observations provide a strong foundation for clinicians 
and researchers to evaluate specific phenotypes of COPD (e.g., frequent ED users) and to develop targeted preventive interventions. For policy makers, our findings underscore the importance of integrated strategies aimed at reducing COPD-related healthcare utilizations in an already-stressed healthcare system.

\section{Abbreviations}

AECOPD: Acute exacerbation of chronic obstructive pulmonary disease; AHRQ: Agency for Healthcare Research and Quality; COPD: Chronic obstructive pulmonary disease; ED: Emergency department; HCUP: Healthcare Cost and Utilization Project; ICD-9-CM: International Classification of Diseases, Ninth Revision, Clinical Modification; SEDD: State Emergency Department Databases; SID: State Inpatient Databases.

\section{Competing interests}

Dr. Camargo has provided consultation for AstraZeneca, Genentech, GlaxoSmithKline, Merck, Novartis, and Pfizer, and received research grants from AstraZeneca and Novartis. The other authors have no financial relationships relevant to this article to disclose.

\section{Authors' contributions}

Conception and design: $\mathrm{KH}, \mathrm{YT}$, and $\mathrm{CC}_{;}$Analysis and interpretation: $\mathrm{KH}, \mathrm{YT}$, $C T, D B$, and $C C$; Drafting the manuscript for important intellectual content: $\mathrm{KH}, \mathrm{YT}, \mathrm{CT}, \mathrm{DB}$, and CC. All authors read and approved the final manuscript.

\section{Funding support}

Dr. Hasegawa was supported, in part, by Eleanor and Miles Shore Fellowship Program (Boston, MA). Dr. Tsugawa was supported, in part, by Honjo International Scholarship Foundation (Tokyo, Japan). The funding organizations had no role in the design and conduct of the study; collection, management, analysis and interpretation of the data; and preparation, review, or approval of the manuscript.

\section{Author details}

1Department of Emergency Medicine, Massachusetts General Hospital, 326 Cambridge Street, Suite 410, Boston, MA 02114, USA. ${ }^{2}$ Harvard Medical School, Boston, MA, USA. ${ }^{3}$ Harvard Interfaculty Initiative in Health Policy, Cambridge, MA, USA.

Received: 19 October 2013 Accepted: 3 April 2014

Published: 10 April 2014

\section{References}

1. Ford ES, Croft JB, Mannino DM, Wheaton AG, Zhang X, Giles WH: Chronic obstructive pulmonary disease surveillance-United States, 1999-2011. Chest 2013, 144:284-305

2. Kanner RE, Anthonisen NR, Connett JE, Group LHSR: Lower respiratory illnesses promote FEV(1) decline in current smokers but not ex-smokers with mild chronic obstructive pulmonary disease: results from the lung health study. Am J Respir Crit Care Med 2001, 164:358-364.

3. Donaldson GC, Seemungal TA, Bhowmik A, Wedzicha JA: Relationship between exacerbation frequency and lung function decline in chronic obstructive pulmonary disease. Thorax 2002, 57:847-852.

4. Seemungal TA, Donaldson GC, Paul EA, Bestall JC, Jeffries DJ, Wedzicha JA: Effect of exacerbation on quality of life in patients with chronic obstructive pulmonary disease. Am J Respir Crit Care Med 1998, 157:1418-1422.

5. The Global Initiative for Chronic Obstructive Lung Disease (GOLD). NHLBI/WHO Gobal Strategy for the Diagnosis, Management and Prevention of COPD. [http://www.goldcopd.org/]

6. Strassels SA, Smith DH, Sullivan SD, Mahajan PS: The costs of treating COPD in the United States. Chest 2001, 119:344-352.

7. Healthy people 2020. [http://www.healthypeople.gov/2020/default.aspx]

8. Hurst JR, Vestbo J, Anzueto A, Locantore N, Mullerova H, Tal-Singer R, Miller B, Lomas DA, Agusti A, Macnee W, Calverley P, Rennard S, Wouters EF, Wedzicha JA: Susceptibility to exacerbation in chronic obstructive pulmonary disease. N Engl J Med 2010, 363:1128-1138.

9. Kim V, Han MK, Vance GB, Make BJ, Newell JD, Hokanson JE, Hersh CP, Stinson D, Silverman EK, Criner GJ, Investigators CO: The chronic bronchitic phenotype of COPD: an analysis of the COPDGene Study. Chest 2011, 140:626-633.

10. Yeatts KB, Lippmann SJ, Waller AE, Lich KH, Travers D, Weinberger M, Donohue JF: Population-based Burden of COPD-related Visits in the Emergency Department (ED): Return ED Visits, Hospital Admissions, and Comorbidity Risks. Chest 2013, 144:784-793.

11. Tsai CL, Griswold SK, Clark S, Camargo CA Jr: Factors associated with frequency of emergency department visits for chronic obstructive pulmonary disease exacerbation. J Gen Intern Med 2007, 22:799-804.

12. Overview of the State Emergency Department Databases (SEDD). Healthcare Cost and Utilization Project. Agency for Healthcare Research and Quality. [http://www.hcup-us.ahrq.gov/seddoverview.jsp]

13. Overview of the State Inpatient Databases (SID). Healthcare Cost and Utilization Project. Agency for Healthcare Research and Quality. [http://www.hcup-us.ahrq.gov/sidoverview.jsp]

14. National Center for Health Statistics: Centers for Disease Control and Prevention. [http://www.cdc.gov/nchs/]

15. Healthcare Cost and Utilization Project Comorbidity Software. [http://www.hcup-us.ahrq.gov/toolssoftware/comorbidity/comorbidity.jsp]

16. Elixhauser A, Steiner C, Harris DR, Coffey RM: Comorbidity measures for use with administrative data. Med Care 1998, 36:8-27.

17. Hasegawa K, Brown DF, Tsugawa Y, Camargo CA Jr: Epidemiology of emergency department visits for opioid overdose: a population-based study. Mayo Clin Proc 2014, 89(4):462-471.

18. Consumer price index. United States Department of Labor, Bureau of Labor Statistics. [http://www.bls.gov/cpi/home.htm]

19. McCullagh P, Nelder JA: Generalized Linear Models (Monographs on statistics and applied probability 37). London: Chapman Hall; 1989.

20. Glynn RJ, Buring JE: Ways of measuring rates of recurrent events. BMJ 1996, 312:364-367.

21. Tsai $\mathrm{CL}$ : Appropriate statistical treatment of frequent emergency department visits in health services research. Ann Emerg Med 2007, 49:385.

22. The Agency for Healthcare Research and Quality: Readmissions for chronic obstructive pulmonary disease. 2008 [http://www.hcup-us.ahrq.gov/ reports/statbriefs/sb121.jsp]

23. Sharma G, Kuo YF, Freeman JL, Zhang DD, Goodwin JS: Outpatient follow-up visit and 30-day emergency department visit and readmission in patients hospitalized for chronic obstructive pulmonary disease. Arch Intern Med 2010, 170:1664-1670.

24. Kim S, Emerman CL, Cydulka RK, Rowe BH, Clark S, Camargo CA, Investigators M: Prospective multicenter study of relapse following emergency department treatment of COPD exacerbation. Chest 2004, 125:473-481.

25. Baillargeon J, Wang Y, Kuo YF, Holmes HM, Sharma G: Temporal trends in hospitalization rates for older adults with chronic obstructive pulmonary disease. Am J Med 2013, 126:607-614.

26. Rabe KF, Wedzicha JA: Controversies in treatment of chronic obstructive pulmonary disease. Lancet 2011, 378:1038-1047.

27. Chiu S, Kelton C, Guo J, Wigle P, Lin A, Szeinbach S: Utilization, spending, and price trends for long-acting beta agonists and inhaled corticosteroids in the US Medicaid program from 1991 to 2010. Am Health Drug Benefits 2011, 4:140-149.

28. Setse RW, Euler GL, Gonzalez-Feliciano AG, Bryan LN, Furlow C, Weinbaum CM, Singleton JA: Influenza Vaccination Coverage-United States, 20002010. MMWR Surveill Summ 2011, 60:38-41.

29. Centers for Disease Control and Prevention: Trends in Current Cigarette Smoking Among high Hschool Students and Adults, United States, 1965-2010. Atlanta, GA: US Department of Health and Human Services; 2011; 2011.

30. Chavez PC, Shokar NK: Diagnosis and management of chronic obstructive pulmonary disease (COPD) in a primary care clinic. COPD 2009, 6:446-451.

31. Donaldson GC, Wedzicha JA: COPD exacerbations .1: epidemiology. Thorax 2006, 61:164-168.

32. Canino G, MCQuaid EL, Rand CS: Addressing asthma health disparities: a multilevel challenge. J Allergy Clin Immunol 2009, 123:1209-1217. quiz 1218-1209.

33. Lange P, Marott JL, Vestbo J, Ingebrigtsen TS, Nordestgaard BG: Socioeconomic Status and Prognosis of COPD in Denmark. COPD 2014 [Epub ahead of print]

34. Prevention quality indicators overview. [http://www.qualityindicators.ahrq. gov/modules/pqi_overview.aspx] 
35. Han MK, Agusti A, Calverley PM, Celli BR, Criner G, Curtis JL, Fabbri LM, Goldin JG, Jones PW, Macnee W, Make BJ, Rabe KF, Rennard SI, Sciurba FC, Silverman EK, Vestbo J, Washko GR, Wouters EF, Martinez FJ: Chronic obstructive pulmonary disease phenotypes: the future of COPD. Am J Respir Crit Care Med 2010, 182:598-604.

36. Population estimates. [http://www.census.gov/popest/]

37. Basu J, Mobley LR: Do HMOs reduce preventable hospitalizations for Medicare beneficiaries? Med Care Res Rev 2007, 64:544-567.

38. Hasegawa K, Tsugawa Y, Brown DF, Mansbach JM, Camargo CA Jr: Temporal trends in emergency department visits for bronchiolitis in the United States, 2006-2010. Pediatr Infect Dis J 2014, 33:11-18.

39. Effing T, Monninkhof EM, van der Valk PD, van der Palen J, van Herwaarden $\mathrm{CL}$, Partidge MR, Walters EH, Zielhuis GA: Self-management education for patients with chronic obstructive pulmonary disease. Cochrane Database Syst Rev 2007(4):CD002990.

40. O'Malley AS, Pham HH, Schrag D, Wu B, Bach PB: Potentially avoidable hospitalizations for COPD and pneumonia: the role of physician and practice characteristics. Med Care 2007, 45:562-570.

doi:10.1186/1465-9921-15-40

Cite this article as: Hasegawa et al.: Frequent utilization of the

emergency department for acute exacerbation of chronic obstructive pulmonary disease. Respiratory Research 2014 15:40.

\section{Submit your next manuscript to BioMed Central and take full advantage of:}

- Convenient online submission

- Thorough peer review

- No space constraints or color figure charges

- Immediate publication on acceptance

- Inclusion in PubMed, CAS, Scopus and Google Scholar

- Research which is freely available for redistribution 